\title{
A NOTE ON A MAXIMAL FUNCTION \\ OF C. FEFFERMAN AND STEIN
}

\section{SAGUN CHANILLO AND RICHARD L. WHEEDEN ${ }^{1}$}

\begin{abstract}
We extend the class of weight functions for which a known inequality for the maximal function $T_{\lambda, r}(f)(x)$ is valid.
\end{abstract}

In this note, we derive a strengthened version of an estimate given by Torchinsky [5] and Barker [1] for the "box" maximal function of C. Fefferman and Stein [3] defined by

$$
T_{\lambda, r}(f)(x)=\sup _{h>0}\left(h^{-\lambda n} \iint_{H(x, h)} t^{\lambda n-n-1}|f(z, t)|^{r} d z d t\right)^{1 / r},
$$

where $1<\lambda<\infty, 0<r<\infty, x$ is a point of $n$-dimensional Euclidean space $R^{n}$, $f(z, t)$ is a function defined in the upper half-space $R_{+}^{n+1}=\left\{(z, t): z \in R^{n}, 0<t<\right.$ $\infty\}$, and $H(x, h)$ is the region $\left\{(z, t) \in R_{+}^{n+1}:|x-z|<h, 0<t<h\right\}$. This maximal function has close connections with the Littlewood-Paley function $g_{\lambda}^{*}($ see $[4,5])$ as well as with Peano maximal functions (see [2]).

We let $B_{h}(x)$ denote the ball in $R^{n}$ with center $x$ and radius $h$, and for $1 \leqslant d<\infty$, consider the class $D_{d}$ of nonnegative measures $\mu$ on $R^{n}$ which satisfy the doubling condition

$$
\frac{\mu\left(B_{h}(x)\right)}{\mu\left(B_{s}(x)\right)} \leqslant c\left(\frac{h}{s}\right)^{n d}, \quad 0<s \leqslant h, x \in R^{n} .
$$

We also define the Hardy-Littlewood maximal function

$$
M_{\mu}(g)(x)=\sup _{h>0}\left(\frac{1}{\mu\left(B_{h}(x)\right)} \int_{B_{h}(x)}|g(z)| d \mu(z)\right),
$$

and the nontangential maximal function

$$
N(f)(x)=\sup _{(z, t) \in \Gamma(x)}|f(z, t)|,
$$

where $\Gamma(x)$ is the cone $\{(z, t):|x-z|<a t\}$ with vertex $x$ and aperture $a$.

We will prove the following theorem.

ThEOREM. Let $\mu \in D_{d}, \lambda \geqslant d, \lambda>1$ and $r>0$. Then

$$
T_{\lambda, r}(f)(x) \leqslant c\left\{M_{\mu}\left(N(f)^{r d / \lambda}\right)(x)\right\}^{\lambda / d r},
$$

with $c$ independent of $f$ and $x$.

Received by the editors August 24, 1982.

1980 Mathematics Subject Classification. Primary 42B25, 42B30.

'Supported in part by NSF grant MCS80-03098. 
This result was proved by Torchinsky [5, Theorem 1] under the additional assumption that $d \mu=w d x$ with $w \in A_{\infty}$ (the class of weight functions introduced by Muckenhoupt and C. Fefferman). We would like to point out that the result in [5] was also proved only in case $\lambda \geqslant d$. In case $w \equiv 1$, the theorem was obtained by Barker [1]; both [1 and 5] generalized an earlier result of Muckenhoupt and Wheeden [4].

The proof we shall give is very similar to those in [ $\mathbf{1}$ and 5], and is based on the following known lemma about Carleson measures. We use the same letter $c$ to denote different constants.

LEMMA. Let $\nu$ and $\sigma$ be nonnegative measures on $R_{+}^{n+1}$ and $R^{n}$ respectively such that $\sigma\left(B_{2 s}(\xi)\right) \leqslant c \sigma\left(B_{s}(\xi)\right)$ for all $\xi \in R^{n}$ and $s>0$. Then for $p_{1} \geqslant p$,

$$
\left(\iint_{R_{+}^{n+1}}|f(z, t)|^{p_{1}} d \nu(z, t)\right)^{1 / p_{1}} \leqslant c\left(\int_{R^{n}} N(f)(z)^{p} d \sigma(z)\right)^{1 / p}
$$

if and only if $\nu(H(\xi, s)) \leqslant c \sigma\left(B_{s}(\xi)\right)^{p_{1} / p}$.

To prove the theorem, fix $h$ and $x$ and write

(1) $\left(h^{-\lambda n} \iint_{H} t^{\lambda n-n-1}|f(z, t)|^{r} d z d t\right)^{1 / r}=\left(\iint_{R_{+}^{n+1}}\left|\chi_{H}(z, t) f(z, t)\right|^{r} d \nu(z, t)\right)^{1 / r}$,

where $H=H(x, h), \chi_{H}$ is the characteristic function of $H$, and $d \nu(z, t)=$ $h^{-\lambda n} t^{\lambda n-n-1} \chi_{H}(z, t) d z d t$. Let $\sigma(\cdot)=\mu(\cdot) / \mu\left(B_{h}(x)\right)$.

We claim that

$$
\nu(H(\xi, s)) \leqslant c\left[\sigma\left(E_{s}(\xi)\right)\right]^{\lambda / d}, \quad \xi \in R^{n}, s>0 .
$$

First note that $\nu(H(\xi, s))=0$ if $H(\xi, s) \cap H=\varnothing$. Also note that

$$
\begin{aligned}
\nu(H(\xi, s)) & =h^{-\lambda n} \iint_{H(\xi, s) \cap H} t^{\lambda n-n-1} d z d t \\
& =h^{-\lambda n}\left(\int_{\substack{0<t<s \\
0<t<h}} t^{\lambda n-n-1} d t\right)\left(\int_{\substack{|z-\xi|<s \\
|z-x|<h}} d z\right) \\
& \leqslant c h^{-\lambda n}(\min \{s, h\})^{(\lambda n-n)+n} \quad(\text { since } \lambda>1) \\
& =c \min \left\{\left(\frac{s}{h}\right)^{\lambda n}, 1\right\} .
\end{aligned}
$$

We distinguish the cases (i) $s<h$ and (ii) $s \geqslant h$. In case (i), we must have $|\xi-x|<2 h$ unless $H(\xi, s) \cap H=\varnothing$. Therefore, $\mu\left(B_{h}(\xi)\right) \sim \mu\left(B_{h}(x)\right)$, and by $D_{d}$ we have

$$
\frac{s}{h} \leqslant c\left[\frac{\mu\left(B_{s}(\xi)\right)}{\mu\left(B_{h}(\xi)\right)}\right]^{1 / n d} \sim\left[\frac{\mu\left(B_{s}(\xi)\right)}{\mu\left(B_{h}(x)\right)}\right]^{1 / n d}
$$


Hence,

$$
\nu(H(\xi, s)) \leqslant c\left(\frac{s}{h}\right)^{\lambda n} \leqslant c\left[\frac{\mu\left(B_{s}(\xi)\right)}{\mu\left(B_{h}(x)\right)}\right]^{\lambda / d} \equiv c \sigma\left(B_{s}(\xi)\right)^{\lambda / d} .
$$

In case (ii), we must have $s \geqslant \frac{1}{2}|\xi-x|$ or else there is no intersection. Therefore, $\mu\left(B_{s}(x)\right) \sim \mu\left(B_{s}(\xi)\right)$. Hence, since also $s>h$,

$$
\nu(H(\xi, s)) \leqslant c \leqslant c\left[\frac{\mu\left(B_{s}(x)\right)}{\mu\left(B_{h}(x)\right)}\right]^{\lambda / d} \leqslant c\left[\frac{\mu\left(B_{s}(\xi)\right)}{\mu\left(B_{h}(x)\right)}\right]^{\lambda / d} \equiv c \sigma\left(B_{s}(\xi)\right)^{\lambda / d} .
$$

This verifies our claim.

By the lemma, since $\lambda \geqslant d,(1)$ is at most

$$
c\left(\int_{R^{n}} N\left(\chi_{H} f\right)(z)^{r d / \lambda} d \sigma(z)\right)^{\lambda / d r} .
$$

Note that $\Gamma(z) \cap H=\varnothing$ unless $z \in B_{(1+a) h}(x)$. Hence,

$$
N\left(\chi_{H} f\right)(z) \leqslant \chi_{B_{(1+u) h}(x)}(z) \cdot N(f)(z),
$$

and (1) is bounded by

$$
c\left(\frac{1}{\mu\left(B_{h}(x)\right)} \int_{B_{(1+u) h}(x)} N(f)(z)^{r d / \lambda} d \mu(z)\right)^{\lambda / d r} .
$$

The theorem follows by taking the supremum over $h$ after observing that $\mu\left(B_{h}(x)\right) \sim$ $\mu\left(B_{(1+a) h}(x)\right)$.

REMARK. If $N_{h}(f)(x)$ denotes the part of the nontangential maximal function with the sup restricted to the truncated cone $\Gamma(x) \cap\{(z, t): 0<t<h\}$, then (2) holds with $N(f)(z)$ on the right replaced by $N_{h}(f)(z)$. Hence, under the hypothesis of the theorem, the proof yields the following estimate, which is more local than that in the theorem:

$$
\begin{aligned}
& \left(h^{-\lambda n} \iint_{H(x, h)} t^{\lambda n-n-1}|f(z, t)|^{r} d z d t\right)^{1 / r} \\
& \quad \leqslant c\left(\frac{1}{\mu\left(B_{h}(x)\right)} \int_{B_{(1+a) h}(x)} N_{h}(f)(z)^{r d / \lambda} d \mu(z)\right)^{\lambda / d r} .
\end{aligned}
$$

We also note in passing that both this estimate and the theorem have analogues for the parabolic metrics in [5].

\section{REFERENCES}

1. S. R. Barker, An inequality for measures on a half-space, Math. Scand. 44 (1979), 92-102.

2. S. Chanillo and R. L. Wheeden, Inequalities for Peano maximal functions and Marcinkiewicz integrals (to appear).

3. C. L. Fefferman and E. M. Stein, $H^{p}$ spaces of several variables, Acta Math. 129 (1972), 137-193. 
4. B. Muckenhoupt and R. L. Wheeden, Norm inequalities for the Littlewood-Paley function $g_{\lambda}^{*}$, Trans. Amer. Math. Soc. 191 (1974), 95-111.

5. A. Torchinsky, Weighted norm inequalities for the Littlewood-Paley function $g_{\lambda}^{*}$, Proc. Sympos. Pure Math., vol. 35, Amer. Math. Soc., Providence, R. I., 1979.

Department of Mathematics, Rutgers University, New Brunswick, New Jersey 08903 (Current address of R. L. Wheeden)

Current address (Sagun Chanillo): Department of Mathematics, Indiana University, Bloomington, Indiana 47405 